\section{Diagnosing narcolepsy}

\section{By Lauren Martz, Staff Writer}

A new way to diagnose narcolepsy using $\mathrm{T}$ cells ${ }^{1}$ has piqued the interest of at least two companies-Jazz Pharmaceuticals plc and GlaxoSmithKline plc. The former could use the results to help diagnose patients eligible for its narcolepsy drug Xyrem sodium oxybate. The latter thinks the findings could help it develop influenza vaccines that lack the extremely rare side effect of inducing narcolepsy.

Narcolepsy causes excessive daytime sleepiness, cataplexy and disruptions in rapid eye movement (REM) sleep. The causes of narcolepsy are unknown but are thought to be a combination of environmental effects and a genetic immune predisposition that causes an autoimmune response against the neurotransmitter orexin (hypocretin; HCRT). The autoimmune attack, in turn, destroys the hypothalamic neurons that produce orexin.

About $98 \%$ of patients with narcolepsy who have an orexin deficiency express the human leukocyte antigen (HLA) molecule DQ0602, whereas only $18 \%-25 \%$ of the general population expresses the molecule. ${ }^{2,3}$

This suggests that antigen presentation by DQ0602 could be associated with an autoimmune response in patients.

Meanwhile, twin studies suggest that the environment is a factor in narcolepsy, as there is only $25 \%-33 \%$ identical twin concordance in the condition. ${ }^{4}$

Another puzzle piece is that some bacterial and viral infections are associated with narcolepsy onset. In 2010, treatment with GSK's pandemic H1N1 influenza vaccine Pandemrix caused an increase in sudden onset narcolepsy in children and adolescents in Europe. ${ }^{5}$ As a result, the EMA's Committee for Medicinal Products for Human Use restricted use of the vaccine in patients under 20 .

In China, the H1N1 flu strain as well as seasonal flu infections also have been linked to new cases of narcolepsy. ${ }^{6}$

Not only is the cause of narcolepsy unclear but also the condition is difficult to diagnose. Current methods include sleep studies, which may be inaccurate, or an invasive procedure to detect orexin levels in cerebrospinal fluid. Diagnosis typically occurs years after symptom onset.

"Narcolepsy is a highly misdiagnosed and underdiagnosed condition," said Jed Black, VP of sleep medicine and a consulting associate professor at the Stanford University School of Medicine's Stanford Center for Sleep Sciences and Medicine. It is estimated that more than $50 \%$ of individuals with narcolepsy have not been diagnosed, he added.

Now, Emmanuel Mignot, Elizabeth Mellins and colleagues may have found a new way to diagnose patients based on the presence of orexinreactive $\mathrm{T}$ cells in the blood. The team also shed light on the role of autoimmunity in narcolepsy pathogenesis.

Mignot is a professor of sleep medicine and of psychiatry and behavior sciences at the Stanford University School of Medicine and director of the Stanford Center for Sleep Sciences and Medicine. Mellins is a professor of pediatrics at Stanford School of Medicine. The paper also included researchers from the University of Bologna, Glostrup Hospital and the Mater Private Sleep Laboratory.

The team first screened overlapping peptide segments of orexin to identify epitopes that bind to and may be presented as antigens by DQ0602. The team identified two epitopes, $\mathrm{HCRT}_{56-68}$ and $\mathrm{HCRT}_{87-99^{2}}$ which bound the narcolepsy-associated HLA molecule and may serve as autoantigens.

The team then engineered antigenpresenting cells to contain only the DQ0602 antigen-presenting molecule and used those cells to present the orexin epitopes to $\mathrm{T}$ cells from patients with narcolepsy and healthy individuals with that HLA molecule.

The assay measured interferon- $\gamma$ (IFNG; IFN- $\gamma$ ) production in response to the antigen presentation and found that only the $\mathrm{T}$ cells from patients were activated by the orexin antigens. - Jed Black, Stanford University School of Medicine

$\mathrm{T}$ cells also reacted to the orexin epitopes from narcoleptic twins or siblings but not from DQ0602+, unaffected siblings. These findings suggest that the assay may be able to specifically diagnose patients with narcolepsy.

The researchers then took their work a step further to determine whether influenza vaccination induces a similar T cell response.

The team vaccinated 9 patients and 4 DQ0602 ${ }^{+}$controls with a 2012 seasonal flu vaccine containing pandemic $\mathrm{H} 1 \mathrm{~N} 1$ antigens and found that the vaccine increased the number of orexin-reactive $\mathrm{T}$ cells in patients but did not affect $\mathrm{T}$ cell reactivity in controls.

The vaccine did not increase narcolepsy symptoms in patients, possibly because orexin was depleted and could not be further decreased by the elevated $\mathrm{T}$ cell activity.

A screen of segments of the hemagglutinin, neuraminidase and polymerase PB1 H1N1-specific viral antigens within Pandemrix may have found the culprit within the vaccine that causes cross-reactivity with orexin-reactive T cells: an epitope of hemagglutinin. The epitope bound DQ0602 and increased the number of both hemagglutinin- and orexin-reactive T cells.

Results were published in Science Translational Medicine.

"A simple blood test would be much easier on the patient, simpler for 
the physician and a substantial reduction on the burden on the healthcare system," said Black.

\section{Diagnostic design}

Black said that new tools such as Stanford's immunoassay would allow physicians or sleep specialists to diagnose the disease more quickly and accurately, which would allow patients to get treatment sooner, although he thinks the Stanford group's diagnostic needs more work before commercialization.

"This data is a long way from a diagnostic product with suitable sensitivity and selectivity to avoid false negatives and false positives," he said.

He added that the Science Translational Medicine paper only discussed narcolepsy patients with cataplexy. "More research would be needed to determine the viability of these diagnostic tools in patients who have narcolepsy without cataplexy," said Black.

Adrian Howd, EVP and head of neurology and corporate development at Evotec AG, agreed that the Stanford team will likely need to improve the sensitivity and specificity of the test.

Evotec has histamine $\mathrm{H} 3$ receptor (HRH3) antagonists including EVT 501 in preclinical testing to treat narcolepsy.

Mignot said that his team's next steps for diagnostic development include working on the sensitivity and specificity of the test. For example, he said, "designing an assay that measures two or three cytokines at the same time or using lower concentrations of the peptide may improve these parameters."

He added that his team also plans to evaluate more samples to determine whether the test can pick up more mild forms of narcolepsy in patients without cataplexy.

Mignot said that "once narcolepsy symptoms develop, 80\%-90\% of the specific neurons are lost. Neurons are really not cells that recover, so when we detect the disease, it is already too late. If this test is able to detect disease in patients at risk or in milder stages of the disease, we may be able to find a way to stop neuron loss, but this application is a long way off."

He added that identifying the disease with this type of assay could be more convenient and accurate than current diagnostic methods and could help patients who have had the disease for a long time get proper treatment.

Jazz Pharmaceuticals said that it is reviewing Mignot's research, as well as research from other labs in the field, to assess the viability of a diagnostic. The company partially funded the work in the Science Translational Medicine paper but has not licensed any related IP.

\section{Influenza implications}

The identification of the Pandemrix component that may trigger $\mathrm{T}$ cell cross-reactivity with self-antigens could lead to the design of safer vaccines.

GSK spokesperson David Daley said, "We are actively conducting research on the observed association between Pandemrix and narcolepsy and on the interaction this vaccine might have had with other risk factors in affected individuals. We hope these ongoing research efforts will enable us to provide more answers."

He said that the new paper "confirms that narcolepsy is a complex disease involving a number of environmental and genetic factors and results from a sequence of these events that we don't yet fully understand. The research suggests a potential mechanism of action that was involved in the onset of narcolepsy in some individuals after vaccination with Pandemrix or infection with H1N1, but the association with Pandemrix remains to be fully explained."

Stanford University has filed a patent application covering the use of orexin epitopes for narcolepsy diagnosis and the modification of the 2009 $\mathrm{H} 1 \mathrm{~N} 1$ hemagglutinin epitope in influenza vaccines. The licensing status is under evaluation.

Martz, L. SciBX 7(3); doi:10.1038/scibx.2014.78

Published online Jan. 23, 2014

\section{REFERENCES}

1. De la Herrán-Arita, A.K. et al. Sci. Transl. Med.; published online Dec. 18, 2013; doi:10.1126/scitranslmed.3007762

Contact: Emmanuel Mignot, Stanford School of Medicine, Stanford, Calif. e-mail: mignot@stanford.edu

Contact: Elizabeth D. Mellins, same affiliation as above e-mail: mellins@stanford.edu

2. Mignot, E. et al. Am. J. Hum. Genet. 68, 686-699 (2001)

3. Han, F. et al. Tissue Antigens 80, 328-335 (2012)

4. Mignot, E. et al. Neurology 50, S16-S22 (1998)

5. Partinen, M. et al. PLoS One 7, e33723; published online March 28, 2012; doi:10.1371/journal.pone.0033723

6. Han, F. et al. Ann. Neurol. 70, 410-417 (2011)

\section{COMPANIES AND INSTITUTIONS MENTIONED}

European Medicines Agency, London, U.K.

Evotec AG (Xetra:EVT), Hamburg, Germany

GlaxoSmithKline plc (LSE:GSK; NYSE:GSK), London, U.K.

Glostrup Hospital, Glostrup, Denmark

Jazz Pharmaceuticals plc (NASDAQ:JAZZ), Dublin, Ireland

Mater Private Sleep Laboratory, Dublin, Ireland

Stanford University, Stanford, Calif.

Stanford University School of Medicine, Stanford, Calif.

University of Bologna, Bologna, Italy 